\title{
A RANDOM INTERVAL GENERATOR USING BETA RAY EMISSION ${ }^{1}$
}

\author{
J. Carmeliet ${ }^{2}$, J. Debecker and John E. Desmedt \\ Brain Research Unit, University of Brussels, 115 boulevard de Waterloo, 1000 Brussels (Belgium)
}

(Accepted for publication: October 29, 1970)

The need for aperiodic stimuli arises in many experimental studies of average sensory evoked potentials. (1) Cerebral evoked potentials may include low voltage components with very long latencies or they may be followed by non-random background activities which are loosely time-locked to the stimulus (Barlow 1960). With stimuli presented at regular intervals such late activities overlap consistently with the early part of the subsequent response, thereby interfering with the averaging process. By contrast, an aperiodic presentation of the stimuli will randomize the phase relations of the overlapping components and, under appropriate conditions, this will improve the efficiency of the computation (Ruchkin 1965), (2) Periodic sensory stimulation at intervals even as long as several seconds is known to modify response wave forms, either by entrainment and recruitment of certain components, or by inducing drop-out or alternation of late components, depending upon their particular recovery cycles. These complex phenomena may be worth studying in their own right, but it is sometimes useful to iron out these special features by using aperiodic stimuli with a mean interval equal to or even shorter than the (fixed) interval considered. (3) In psycho-physiological studies stimuli delivered at constant intervals or in regular alternation int roduce a bias in the subject's decision task and they also influence the cerebral evoked potential wave form significantly ( $c f$. Walter et al. 1964), since each stimulus of the sequence provides a warning of the next. By using random intervals between stimuli or by programming successive uni- or pluri-modal stimuli into random sequence, such interference related to the subject's anticipation or expectancy can be practically eliminated (Debecker and Desmedt 1970).

Random programs have been rather little used in evoked potential studies, apparently because suitable designs or commercial equipment were not available. Mechanical contactors (Funke et al. 1969), punched tape

1 The research reported has been supported by the National Institute of Neurological Diseases and Stroke, National Institutes of Health, U.S. Public Health Service, by the Office of Naval Research, U.S. Navy, and by the Belgian Fonds de la Recherche Scientifique Mèdicale.

"From the Laboratoire de Métrologie nucléaire, University of Brussels. Requests for reprints should be addressed to the Brain Research Unit. loops or magnetic tape recorders can be made to deliver electric pulses according to a built-in schedule of intervals which has been pre-recorded with the help of a random number list. The electronic noise of a tube or a diode can be amplified and added to near-threshold pulses to produce intermittent triggering in an electronic circuit (Etter 1966). If the laboratory includes a general purpose computer this can of course provide a sophisticated source of random numbers which can be converted into intervals by a count-down technique.

This paper describes an easily built and rugged solid state generator of random intervals which has been in use for several years and which provides a most convenient item to include in any electronic programming system ${ }^{3}$.

\section{MODE OF OPERATION}

The instrument uses a weak beta ray emitter formed by deposition of ${ }^{90} \mathrm{Sr}$ (half life 28 years) onto an aluminium disc. The emitter is placed in a lead enclosure with a $1 \mathrm{~mm}$ diameter opening through which the electrons reach a 0.4 $\mathrm{mm}$ thick plastic scintillator sheet (NE-102, Nuclear Enterprise) (Fig. 1, A). The collision between an electron and an atom in the plastic sheet triggers an emission of photons, the number of which varies with the kinetic energy transferred during the corresponding impact. An interesting feature of the beta emission is that the spectrum of this kinetic energy is continuous from zero to a maximum level which is characteristic of the radionuclide used. The measured spectrum shows a peak incidence of energy levels close to the minimum, while the incidence decreases regularly for higher and higher energy levels and becomes zero for the maximum energy level ( $c f$. Evans 1955). The kinetic energy, and hence the number of photons emitted in the plastic scintillator, varies at random from one disintegration to the next.

The scintillator is placed on the window of an EMI 5924 photomultiplier tube operated at $-600 \mathrm{~V}$, obtained

${ }^{3}$ Since this paper was submitted for publication we have become aware that Mr. H. B. Morton of the National Hospital, Queen Square, London, has been using beta emission for the same purpose for some years. His device uses a Geiger counter and the mean pulse rate is controlled simply by varying the distance between the beta source and the counter. 

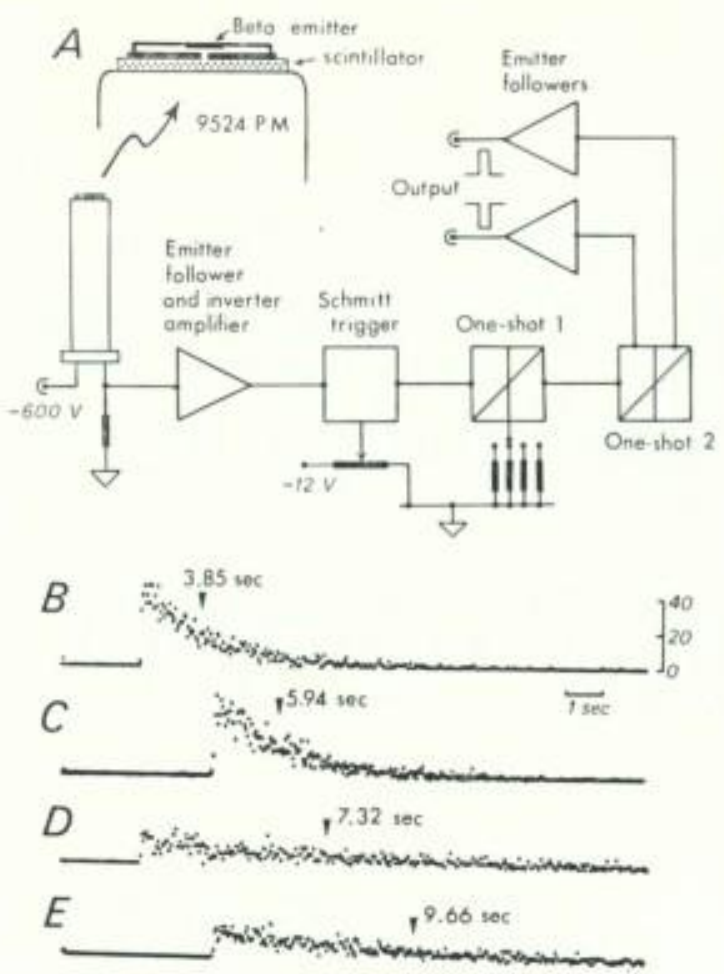

Fig. 1

$A$, block diagram of the generator of random intervals with a detailed sketch (to scale) of the arrangement of the beta emitter chamber. $B-E$, time histograms of intervals between 2000 successive output pulses, for various settings of the generator controls. The ordinate represents the number of intervals of any duration (bin width $=25 \mathrm{msec}$ ).

from 7 voltage stabilizer tubes $85 \mathrm{~A} 2$. Each emission of photons thus results in a brief current pulse in the anode resistance of the photomultiplier. This current is proportional to the number of photons emitted by the collision and hence to the energy of the corresponding electron ejected by the ${ }^{90} \mathrm{Sr}$. The photomultiplier output is connected through emitter follower and amplifier stages to an NPN transistor Schmitt trigger (Fig. 2, $T_{4-5}$ ), the threshold of which is adjusted by a potentiometer in the base circuit of $\mathrm{T}_{4}$. If a low threshold is set in the Schmitt circuit most of the pulses from the photomultiplier will trigger and the proportion of brief (random) intervals will increase (Fig. 1. $B$ and $C$ ). If a higher threshold is set by the potentiometer the trigger will only flip for the larger pulses which correspond to the emissions triggering a larger number of photons. In view of the configuration of the energy spectrum just discussed, the triggering level will in fact permit a continuous adjustment of the mean frequency of the random pulses (Fig. 1, $D$ and $E$ ).

The next stage is a one-shot multivibrator with several pre-set recovery times from 1 to $5 \mathrm{sec}$ (Fig. 2, $\mathrm{T}_{\mathrm{i}-\mathrm{i}}$ ). This serves to exclude pulses arriving from the Schmitt trigger before a chosen minimal interval after the preceding pulse (compare Fig. 1, B and $C$ ). Other dead time intervals can be selected by adding the appropriate resistors in the rotary switch. The next stage $T_{t-9}$ is another one-shot which shapes the output square pulses delivered via emitter followers $T_{10}$ and $T_{11}$. Low impedance positive and negative pulses of $50 \mu \mathrm{sec}$ duration and $10 \mathrm{~V}$ are thus available.

The circuit of Fig. 2 follows conventional design and includes 11 transistors of which 9 are of the NPN type. Note that the emitter follower $T_{1}$ is fed by current flowing in the series of resistors providing the voltage levels for the 11 dynodes of the photomultiplier tube. The collector voltage of $T_{1}$ is stabilized by an electrolytic condenser.

\section{DISCUSSION}

Nuclear processes such as beta emission have a truly random characteristic ( $c f$. Evans 1955) and this feature has been used for designing a convenient and reliable random interval generator suitable for electrophysiological programming. The unit described requires no special precautions for radiation protection since it uses a weak radionuclide enclosed in lead. Its performance is unaffected by temperature changes.

Any range of intervals can easily be provided by the unit. The mean rate of collision in the plastic scintillator can be reduced to the practically useful range by collimation, $i, e .$, by adjusting the size of the hole in the lead enclosure. Unless other conditions are required, this adjustment need not be repeated since the radionuclide has a half life as long as 28 years. When the instrument is in use a simple potentiometric control allows the selection of the appropriate mean of random intervals. Fig. 1 illustrates time histograms of intervals between 2000 consecutive output pulses from the unit, as recorded and displayed by a Mnemotron CAT B computer in the histogram mode. Two different settings of the potentiometer in $T_{4}$ base were used for recording B-C and D-E, respectively. With a higher treshold of $T_{4}$ there are many more large intervals and there is an increase in the mean, which is indicated on each histogram by an arrow. However, some preponderance of the smaller intervals still appears in the distribution at the higher setting shown. Such a skewed distribution of the random intervals is indeed convenient in many physiological applications because it avoids unduly lengthening the mean interval, and hence the duration of the experimental runs, while preserving the aperiodic nature of the stimulation sequence. In any case it is possible to obtain, if required, very dispersed interval distributions which approximate a rectangular distribution by increasing the threshold of $T_{4}$. An independent control of the minimal interval between suecessive output pulses is provided by a rotary switch controlling the recovery time of the $\mathrm{T}_{6-7}$ one-shot.

The generator can be used in several ways, for example to trigger stimulators and deliver a sensory stimulus at random intervals. It can also open and close gate circuits in order to provide random sequences of different events or stimuli.

Electroenceph clin. Neurophysiol., 1971, 30: 354-356 


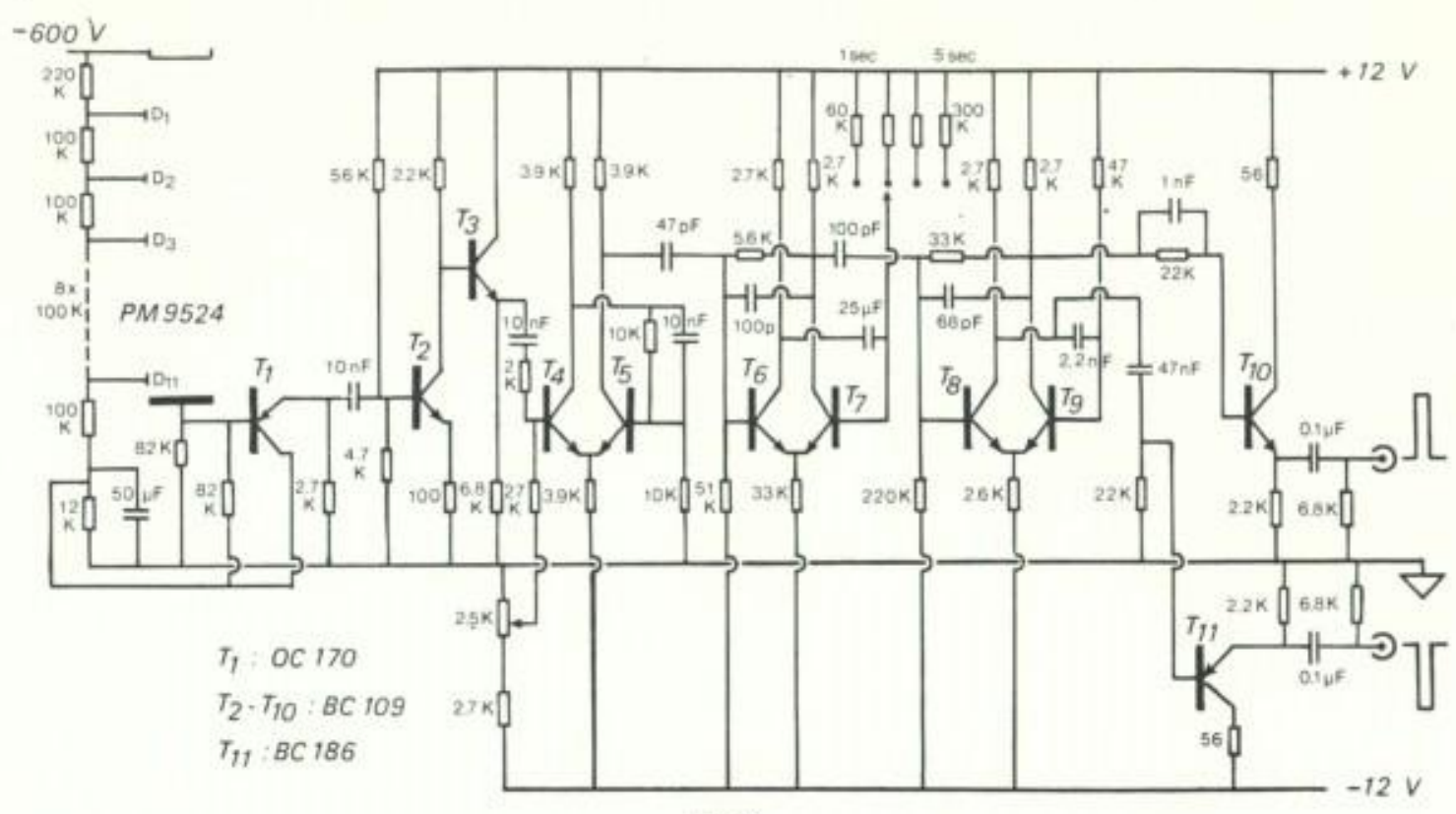

Fig. 2

Circuit of the generator of random intervals.

\section{SUMMARY}

A solid state generator of random intervals, based on a beta ray emitter, is described. The instrument provides for independent control of the minimal interval between successive output pulses and of the dispersion of the random intervals. It has been used for delivering sensory stimuli at random intervals or in random sequences.

\section{RESUME}

\section{GENERATEUR D'INTERVALLES ALEATOIRES}

\section{UTILISANT UNE SOURCE RADIOACTIVE BETA}

Le travail décrit un générateur transistorisé d'intervalles aléatoires qui utilise une source radioactive béta. L'instrument permet de contrôler indépendamment. l'intervalle minimal entre deux impulsions de sortie successives, et la dispersion des intervalles aléatoires autour de la moyenne. Il a été utilisé pour déclencher des stimulations sensorielles à intervalles aléatoires, ou selon une séquence irrégulière.

\section{REFERENCES}

BARLOW, J, S. Rhythmic activity induced by photic stimulation in relation to intrinsic alpha activity of the brain in man. Electroenceph. clin. Neurophysiol., 1960, 12: 317-326.

Debecker, J. and Desmedt, J. E. Maximum capacity for sequential one-bit auditory decisions. J.exp. Psychol., $1970,83: 366-372$.

ETtER, T. L. A random stimulus generator. Psychophysiology, 1966, 2: 384-387.

Evans, R. D. The atomic nucleus. Mc Graw Hill, New York, 1955, 972 p.

FunKE, E. R., Sнеwсhuк, A. and James, E. Low-cost pseudo random pulse generator. Med. Res. Engineer, $1969,8: 43-44$.

RuCHKIN, D. S. An analysis of average response computations based upon aperiodic stimuli. IEEE Trans. biomed. Engin., 1965, BME-12: 87-94.

Walter, W. G., COOPER, R., Aldridge, V.J., McCallum, W. C. and WINTER, A. L. Contingent negative variation: an electric sign of sensori-motor association and expectancy in the human brain. Nature (Lond.), 1964, 203: $380-384$.

Reference: CARmeliet, J., Deheckfr, J. and Desmedt, J. E. A random interval generator using beta ray emission. Electroenceph. clin. Neurophysiol., 1971, 30: 354-356. 